\title{
Defining the nociceptor transcriptome
}

\section{Matthew Thakur ${ }^{1+}{ }^{+}$, Megan Crow ${ }^{1+}$, Natalie Richards ${ }^{1+}$, Gareth I. J. Davey ${ }^{1}$, Emma Levine ${ }^{1}$, Jayne H. Kelleher ${ }^{1}$, Chibeza C. Agley ${ }^{2}$, Franziska Denk ${ }^{1}$, Stephen D. R. Harridge ${ }^{2}$ and Stephen B. McMahon ${ }^{1}$}

${ }^{1}$ Wolfson Centre for Age-Related Diseases, King's College London, London, UK

${ }^{2}$ Centre of Human and Aerospace Physiological Sciences, King's College London, London, UK

\section{Edited by:}

Bernard Attali, Tel Aviv University,

Israel

Reviewed by:

Terrance P. Snutch, The University of British Columbia, Canada

Nikita Gamper, University of Leeds, UK

*Correspondence:

Matthew Thakur, Wolfson Centre for

Age-Related Diseases, King's

College London, Guy's Campus,

London SE1 1UL, UK

e-mail: stephen.mcmahon@kcl.ac.uk

${ }^{\dagger}$ These authors have contributed equally to this work.
Unbiased "omics" techniques, such as next generation RNA-sequencing, can provide entirely novel insights into biological systems. However, cellular heterogeneity presents a significant barrier to analysis and interpretation of these datasets. The neurons of the dorsal root ganglia (DRG) are an important model for studies of neuronal injury, regeneration and pain. The majority of investigators utilize a dissociated preparation of whole ganglia when studying cellular and molecular function. We demonstrate that the standard methods for producing these preparations gives a 10\%-neuronal mixture of cells, with the remainder of cells constituting satellite glia and other non-neuronal cell types. Using a novel application of magnetic purification, we consistently obtain over 95\% pure, viable neurons from adult tissue, significantly enriched for small diameter nociceptors expressing the voltage gated ion channel $\mathrm{Na}_{v}$ 1.8. Using genome-wide RNA-sequencing we compare the currently used (10\% neuronal) and pure (95\% nociceptor) preparations and find 920 genes enriched. This gives an unprecedented insight into the molecular composition of small nociceptive neurons in the DRG, potentially altering the interpretation of previous studies performed at the tissue level, and indicating a number of novel markers of this widely-studied population of cells. We anticipate that the ease of use, affordability and speed of this technique will see it become widely adopted, delivering a greatly improved capacity to study the roles of nociceptors in health and disease.

Keywords: pain, nociceptors, nociception, somatosensation, dorsal root ganglion, RNA-sequencing, peripheral nervous system, regeneration

\section{INTRODUCTION}

Cell sorting techniques combined with transcriptional or epigenetic profiling provide a unique insight into how gene expression is regulated across different cell types. While cell type-specific profiling is now widely employed by researchers in the oncology and immunology fields (Hu et al., 2005; Heng et al., 2008), most researchers studying the nervous system continue to assess gene expression at the whole tissue level.

This is despite the fact that neural tissues contain a wide variety of different cell types such as epithelial cells, fibroblasts, glia and macrophages, as well as molecularly and functionally distinctive subsets of neurons. This heterogeneity significantly limits the usefulness of transcriptomic and epigenetic data generated to date. While sorting techniques, including fluorescenceactivated cell sorting (FACS), have been applied to neural tissue, these often require transgenic animals and large amounts of cellular material in order to recover sufficient RNA, and are often better suited to neonate than adult neural tissue (Lobo et al., 2006).

In the peripheral nervous system, a commonly studied tissue is dorsal root ganglion (DRG). The peripheral nerve cell bodies contained in the DRG are important targets for investigators assessing sensory function, injury, regeneration and pain. Chronic pain is one of the leading causes of long term disability, estimated to affect one in five adults (Breivik et al., 2013). More than one in three patients suffering chronic pain report inadequate pain relief (Breivik et al., 2013) — yet many promising preclinical drug targets have not translated into effective treatments. One reason for this is the relatively poor understanding of the fundamental physiology and pathophysiology of pain-sensing nerve fibers (nociceptors, a subset of peripheral nerve cells) at the molecular level (Eijkelkamp et al., 2012).

We present a novel application of magnetic cell sorting (MACS) which generates a 95\% pure nociceptor preparation from adult mouse DRG. This MACS preparation is enriched for small nociceptive neurons expressing the voltage gated sodium channel $\mathrm{Na}_{\mathrm{v}} 1.8$. Comparison of gene expression in mixed dissociated ganglion with the MACS purified nociceptor preparation reveals the transcriptional signature of nociceptors.

\section{MATERIALS AND METHODS \\ ANIMALS}

Adult male C57Bl/6 mice weighing between 20 and $25 \mathrm{~g}$ were used for all experiments (Harlan, Bicester, UK). Animals were housed under standard conditions $(12 \mathrm{~h}$ light/dark cycle, lights on between 7:00 a.m. and 7:00 p.m., free access to lab chow and water, groups of 4-8). All care of animals was in accordance with the United Kingdom Animals Scientific Procedures Act (1986). $\mathrm{Na}_{\mathrm{v}} 1.8$ TdTomato mice have been extensively characterized elsewhere (Shields et al., 2012). 


\section{TISSUE PREPARATION}

Mice were euthanized with a terminal dose of pentobarbital and decapitated. DRG were taken from all levels as previously described (Malin et al., 2007). For experiments on intact ganglia, DRG were snap frozen using liquid nitrogen, before being homogenized in Trizol (Invitrogen). For unsorted and MACSsorted preparations, DRG were washed in F12 medium followed by incubation in dissociating enzymes $(3 \mathrm{mg} / \mathrm{mL}$ dispase, $0.1 \%$ collagenase and $200 \mathrm{U} / \mathrm{ml}$ DNAse, all from Roche) for $30 \mathrm{~min}$ at $37^{\circ} \mathrm{C}$. Dissociation enzymes were then removed and replaced with F12. Cells were triturated 5 times using a P1000 tip to disrupt the tissue. Cells in suspension were retained, and fresh F12 was added to the remaining undisrupted material. This process was repeated 5 times. Cells in suspension were filtered through a $40 \mu \mathrm{m}$ filter and centrifuged for $5 \mathrm{~min}$ at $150 \mathrm{G}$. For unsorted preparations, cells were resuspended in Trizol at this point and snap frozen.

\section{MACS-SORTING OF DISSOCIATED DORSAL ROOT GANGLIA}

Dissociated DRG neurons were washed in Dulbeccos PBS then resuspended in $120 \mu \mathrm{l}$ MACS buffer (Miltenyi autoMACS HBSSbased washing solution with $0.5 \%$ bovine serum albumin). $30 \mu \mathrm{l}$ of biotinylated non-neuronal antibody cocktail was added (Miltenyi MACS Neuron Isolation Kit) and cells incubated at $4^{\circ} \mathrm{C}$ for $5 \mathrm{~min}$. After washing in MACS buffer, cells were resuspended in $120 \mu \mathrm{l}$ MACS buffer with $30 \mu \mathrm{l}$ anti-biotin microbeads (Miltenyi MACS Neuron Isolation Kit) and incubated at $4{ }^{\circ} \mathrm{C}$ for $10 \mathrm{~min}$. Cells were then run through a LD exclusion column placed in a QuadroMACS separator (Miltenyi Biotech), which retained all microbead-conjugated, non-neuronal cells so that only neurons were eluted. For MACS-sorted preparations, eluted pure neurons were then centrifuged, resuspended in Trizol and snap frozen.

\section{IMMUNOCYTOCHEMISTRY}

For characterization, unsorted preparations were plated on poly-L-lysine/laminin coated coverslips. Sorted cells were plated on poly-L-lysine coverslips incubated with Matrigel (BD Biosciences) diluted 1:10 in F12. After $\sim 18 \mathrm{~h}$ in vitro, cells were fixed in $2 \% \mathrm{PFA}$ at $37^{\circ} \mathrm{C}$ for $10 \mathrm{~min}$. Cells were then blocked in $10 \%$ Normal Donkey Serum in PBS with $0.2 \%$ Triton-X for $30 \mathrm{~min}$ at room temperature, followed by a $30 \mathrm{~min}$ RT incubation in primary antibody. Cells were washed in PBS and incubated in secondary antibody at room temperature for $30 \mathrm{~min}$, followed by further washing and coverslipping with Vectashield mounting medium (Vector Labs). Primary antibodies used were: mouse $\beta$-III tubulin (Promega, G712A), rabbit glutamine synthetase (Abcam, ab498371), mouse CGRP (Abcam, ab81887). Secondaries were all Alexa 488 or 546 (Life Technologies). Fluorescence was visualized with fixed exposure times using a Zeiss Axioplan 2 microscope and analyzed using ImageJ software and Graphpad Prism. Positive cells were defined as cells with an intensity $>30 \%$ that of the mean top 10 most intense cells in the image being analyzed.

\section{FLOW CYTOMETRY OF DISSOCIATED NEURONAL PREPARATIONS}

Cells were prepared for cytometry as described above, with two variations. Cells were prepared in cytometry buffer instead of medium (Hank's Buffered Saline Solution with 1 M HEPES and 0.5 M EDTA). Cells were prepared from mice expressing GFP in Advillin-positive cells, i.e., all DRG neurons (Advillin-GFP mouse obtained from Gensat). The pan-nuclear marker DRAQ5 (Biostatus, UK) was then added at 1:10,000. Unsorted and MACS sorted samples were analyzed using an LSR Fortessa. The number of GFP-positive events were quantified as a percentage of all DRAQ5-positive events to assay the proportion of neuronal to non-neuronal cells.

\section{RNA EXTRACTION AND LIBRARY PREPARATION FOR SEOUENCING}

RNA was extracted from intact, unsorted and MACS-sorted samples using the RNeasy micro kit (Qiagen). Each sample (each $n$ ) comprised material from 1, 2 or 8 mice in the intact, unsorted and MACS-sorted groups, respectively. RNA integrity was assessed on an Agilent2100 Bioanalyzer (Agilent, Santa Clara, CA) and the RNA integrity number of each sample was $>8$. PolyA-selected RNA was used for complimentary DNA library preparation using the NEBNext Ultra direction RNA Library Prep Kit according to manufacturer's instructions (NEB, Ipswich, MA). 100 bp reads were sequenced on the Illumina HiSeq2000/2500 platform in replicate flow cells.

\section{DATA ANALYSES}

Quality control, alignment and differential expression analyses were performed on the Galaxy server (Giardine et al., 2005; Blankenberg et al., 2010; Goecks et al., 2010). Cortical neuron fastQ files were uploaded to Galaxy directly from the European Nucleotide Archive (http://www.ebi.ac.uk/ena/ data/view/SRP033200, sample accessions SAMN02415125, SAMN02415124). Briefly, fastQ files were uploaded, assessed for sequencing quality using fastQC (http://www.bioinformatics. babraham.ac.uk/projects/fastqc) and Picard Alignment Summary Metrics (http://picard.sourceforge.net). Base calls were of high quality across the full-length of the reads and trimming was not required. FastQ files were aligned to the UCSC mm10 build of the mouse genome using TopHat2 (Kim et al., 2013), with the multiple alignment parameter (-g) set to two. Aligned bam files from separate lanes were combined using the Merge Bam tool from SamTools (Li et al., 2009), then fragments per kilobase per million (FPKM) estimates were obtained using Cufflinks version 2.1.1 (Trapnell et al., 2012). Genes were considered to be expressed if more than three samples within a group had an FPKM value greater than 5.

Differential expression analyses were performed using CuffDiff version 2.1.1 (Trapnell et al., 2012). Genes were considered to be differentially expressed if they had a $q<0.05, \log 2$-fold change $<-0.5$ or $>0.5$, and mean FPKM $>5$ in the group with higher expression. Following differential expression analysis of MACS sorted samples it became clear that these were contaminated with red blood cells (e.g., they had high levels of canonical blood genes, such as $H b b-b s)$. To account for this, all genes that were found to be both significantly upregulated in MACS samples and had a median $\log 2 \mathrm{RPKM}>3$ in a recently published mouse erythrocyte transcriptome study (An et al., 2014) were removed prior to subsequent analysis (totaling 308 blood genes). 
Principal component analysis, density and scatter plots were created using the cummeRbund package in $\mathrm{R}$ (version 3.1.0). Hierarchical clustering and heat maps were generated using MultiExperiment Viewer (MeV) (Saeed et al., 2003). DAVID (Da Wei Huang and Lempicki, 2008; Huang et al., 2009), Panther (Mi et al., 2013), and STRING (Jensen et al., 2009) were used for gene functional annotation and network analyses.

Datasets are available via GEO accession number GSE62424.

\section{RESULTS \\ MACS-SORTED PURE NEURONAL PREPARATIONS ARE ENRICHED FOR NOCICEPTORS}

We used a novel application of magnetic cell sorting (MACS) to obtain a 95\% pure peripheral nerve cell body preparation from adult DRG. Quantification of traditionally prepared, unsorted dissociated DRG cultures stained for the neuronal marker $\beta$-III Tubulin, the satellite glial cell marker glutamine synthetase (GS) and the nuclear marker DAPI demonstrate that neuronal cells constitute a mean of $9 \%$ of adherent, nucleated cells present in these preparations one day after dissociation (Figures 1A,C). In contrast, a DRG preparation subjected to MACS contains more than 95\% neuronal nuclei (Figures 1B,C). Cell size distribution indicates that magnetic sorting generates a preparation enriched for cells $<30 \mu \mathrm{m}$ in diameter (nociceptors) (Figure 1D).

Flow cytometry analysis of acutely dissociated DRG preparations confirms that neurons constitute $9.8 \%$ of nucleated events in the acute preparation (Supplementary Figure 1). This indicates that the relatively large proportion of non-neuronal cells detected after 1 day in culture do not simply reflect proliferating cells, but is an accurate reflection of the cellular makeup of the acute preparation. Note that while we observed that neurons only constituted $\sim 10 \%$ of cells in acutely dissociated preparations of DRG, this is likely an underestimate of the total proportion of neurons in the intact DRG as the dissociation process will cause some cells, such as larger DRG neurons, to lyse.

We next performed MACS sorting on dissociated DRG from transgenic animals carrying the TdTomato reporter in cells expressing the voltage-gated sodium channel, $\mathrm{Na}_{\mathrm{v}} 1.8$ (Figures 1E,F). This confirmed that the MACS sorting process generates a cell preparation enriched for nociceptors. In agreement with previous studies (Shields et al., 2012), $\mathrm{Na}_{\mathrm{v}} 1.8+$ cells make up only $76 \%$ of dissociated unsorted neurons, yet constitute 95\% of the MACS sorted preparation (Figure 1G).

The proportion of cells expressing the neuropeptide calcitonin gene-related peptide (CGRP), which defined a subset of nociceptors, is similar in unsorted (Figure 1H, 38\%) and sorted preparations (Figure 1I, 36\%), indicating that the MACS sorting process enriches cells according to size but introduces no bias in neurochemical subtype (Figure 1J).

\section{RNA-SEQUENCING OF INTACT, DISSOCIATED AND MACS-SORTED PREPARATIONS IDENTIFIES THE NOCICEPTOR TRANSCRIPTOME}

We next extracted high-quality RNA from either whole, intact DRG, acutely dissociated, unsorted DRG or MACS-sorted DRG for use in RNA-sequencing (study design illustrated in schematic Figure 2A). The sequencing data was used to identify unbiased gene expression profiles across the entire transcriptome. Sequencing was performed on polyA + messenger RNA of four biological replicates for each condition at a depth of $\sim 18$ million reads per sample. Sequencing data were subject to quality control analyses prior to alignment with TopHat2. A high-confidence transcriptome was generated using Cufflinks in Galaxy, and differences in gene expression levels were analyzed using CuffDiff. Only annotated genes were analyzed.

We initially determined how many genes were expressed in all three conditions. We detected 7987 genes in intact DRG, 7929 genes in dissociated, unsorted samples and 7309 genes in MACSsorted samples, corresponding to $\sim 30 \%$ of known murine genes (Figure 2B). The vast majority of these genes $(6876,81 \%)$ were expressed in all three groups (Figure 2C).

Cell-type enrichment analysis performed using the CTen platform (Shoemaker et al., 2012) accurately identified the overlapping genes as DRG and other neuronal cell types (Figure 2D). In contrast, genes found only in the intact and dissociated groups were not identified as neuronal, confirming the ability of the MACS sorting process to exclude non-neuronal cells.

Principal component analysis (PCA) was performed on all genes. Intact DRG and dissociated, unsorted samples clustered closely together, while MACS-sorted samples clustered separately (Figure 2E). Pairwise comparison of replicates within each group revealed a high degree of correlation $(\mathrm{r} 2=0.81-0.87)$, indicating the reproducibility of the preparation methods.

\section{THE MACS-SORTED CELL TRANSCRIPTOME IDENTIFIES NOVEL NOCICEPTOR-ENRICHED ION CHANNEL GENES}

Nociceptor genes were identified by comparing MACS-sorted and unsorted DRG preparations using the CuffDiff algorithm. A total of 2392 differentially expressed genes were discovered, of which $920(38.5 \%)$ were enriched in the MACS-sorted samples and 1473 (61.5\%) were depleted (Figure 3A).

Consistent with the in vitro characterization, genes enriched in the MACS-sorted samples include many known small neuronal markers such as GDNF responsive receptor tyrosine kinase, Ret $(\log 2$-fold change $=0.79, q=0.0002)$ and neurofilament peripherin (Prph log2-fold change $=0.39, q=0.011)$. Depleted genes include the large neuronal marker parvalbumin (log2-fold change $=-3.34, q=0.0002)$ and the glial cell glutamate transporter GLAST-1 (Slc1a3, log2-fold change $=-6.99, q=0.0044)$. The genes enriched in MACS-sorted neurons represent all genes expressed more abundantly in nociceptors than in other DRG neurons and non-neuronal cells. To determine whether these genes may also be nociceptor-specific, we performed an in silico analysis of MACS transcriptional data against global gene expression data from pure cortical neurons (Zhang et al., 2014). 6140 genes were found to be differentially expressed between the two datasets, of which 3190 (52\%) were enriched in cortical neurons and 2950 (48\%) were enriched in nociceptors (described in Supplementary Figure 2 and Supplementary Table 2). Of the 920 genes most enriched in the MACS-sorted preparation compared to unsorted DRG, 548 (68\%) were also significantly enriched compared to cortical neurons. This suggests that these genes are not only nociceptor-enriched, but may in fact be nociceptorspecific. 

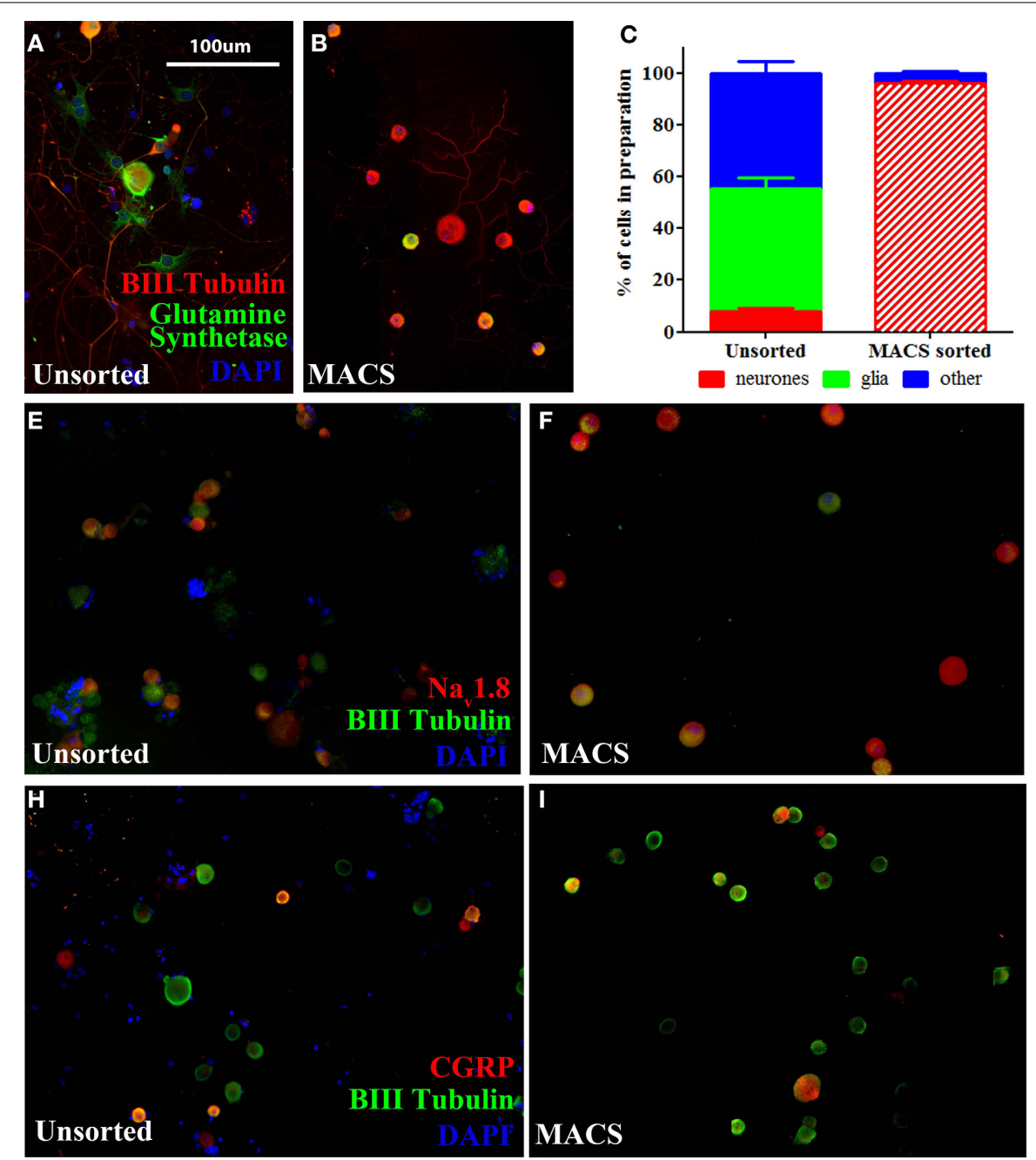

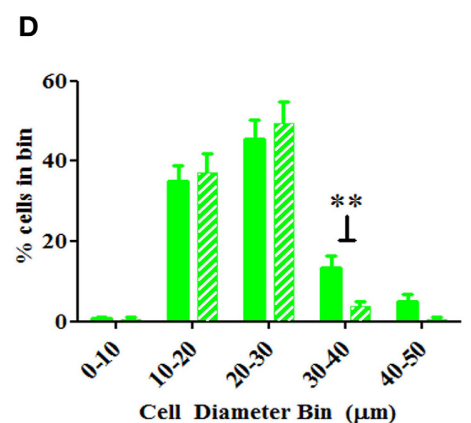

Cell Diameter Bin $(\mu \mathrm{m})$

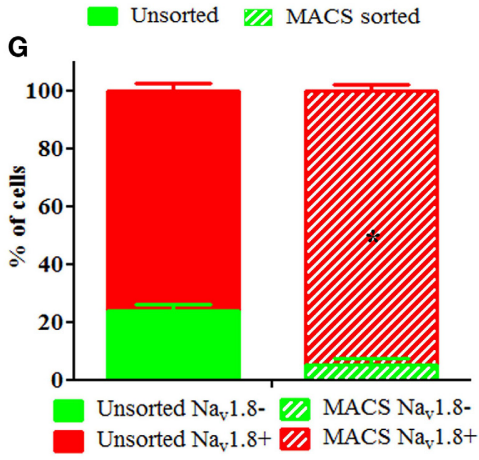

$\mathbf{J}$

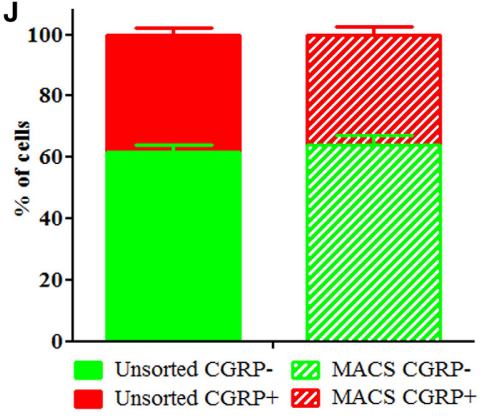

FIGURE 1 | Characterisation of MACS-sorted DRG neuronal preparation. (A,B) Immunohistochemistry visualizing neuronal ( $\beta$-III tubulin), satellite glial (glutamine synthetase) or other non-neuronal (DAPI) cells in unsorted and MACS-sorted neuronal preparations. (C) Quantification of neuronal, glial and other non-neuronal cells shows that MACS-sorting generates $>95 \%$ pure neuronal preparation ( $n=8$ per condition). (D) Cell size distribution of neuronal cells in unsorted and MACS-sorted preparations shows that the MACS-sorted preparation is enriched for small cells $<30 \mu \mathrm{m}$ in diameter $(n=8 \mathrm{per}$ condition. $^{* *}=$ unpaired $t$-test $\left.p<0.01\right)$. (E,F) Immunohistochemistry of unsorted and MACS-sorted preparations generated from Nav1.8 TdTomato reporter mice. (G) Percentage of cells positive for $\mathrm{Na}_{\vee} 1.8$ is significantly greater following MACS sorting ( $n=4$ per condition, Fisher's exact test, $\left.{ }^{*} p<0.05\right)$. $(\mathbf{H}, \mathbf{I})$ Immunohistochemistry visualizing the neuropeptide calcitonin gene related peptide (CGRP) in unsorted and MACS-sorted preparations. (J) The percentage of cells expressing CGRP is unchanged in MACS-sorted preparations ( $n=4$, Fisher's exact test $p=0.229$ ).
Table 1 below shows highlighted results. All MACS vs. DRG values are accessible in Supplementary Table 1 and MACS vs. Cortical Neurons may be found in Supplementary Table 2.

Functional annotation clustering using DAVID indicates that genes enriched in MACS-sorted preparations possess GO terms related to ion channel activity and ion binding (false discovery rate corrected $p$-value range $6.55 \mathrm{e}-4-2.8 \mathrm{e}-7$; Supplementary Table 3), and 43 genes related to ion channel activity were identified for downstream analysis (Figure 3B).

Jamieson et al. (2014) have recently described the pain interactome, a pain-specific protein-protein interaction network (Jamieson et al., 2014). Using the key nodes of the pain interactome alongside the 43 ion channel activity genes in an unbiased network analysis we discovered two major gene clusters
(Figure 3C). The largest cluster contained the known painrelated genes from the pain interactome as well as various transient receptor potential channel members, purinoreceptors and voltage-gated sodium channel subunits, the majority of which have known functions in somatosensation or nociception. The second cluster primarily contained calcium and potassium channel subunits, including the calcium channel subunit Cacna2d1 (alpha-2-delta-1) which is the major target of the neuropathic pain drug, gabapentin (Bauer et al., 2009). The remaining ion channel activity-related genes did not form clusters as they had no known interactions with either the pain-related genes or the calcium and potassium channel subunits, therefore representing a novel subset of nociceptor-enriched ion channel genes, the majority of which have not been previously linked to pain. 


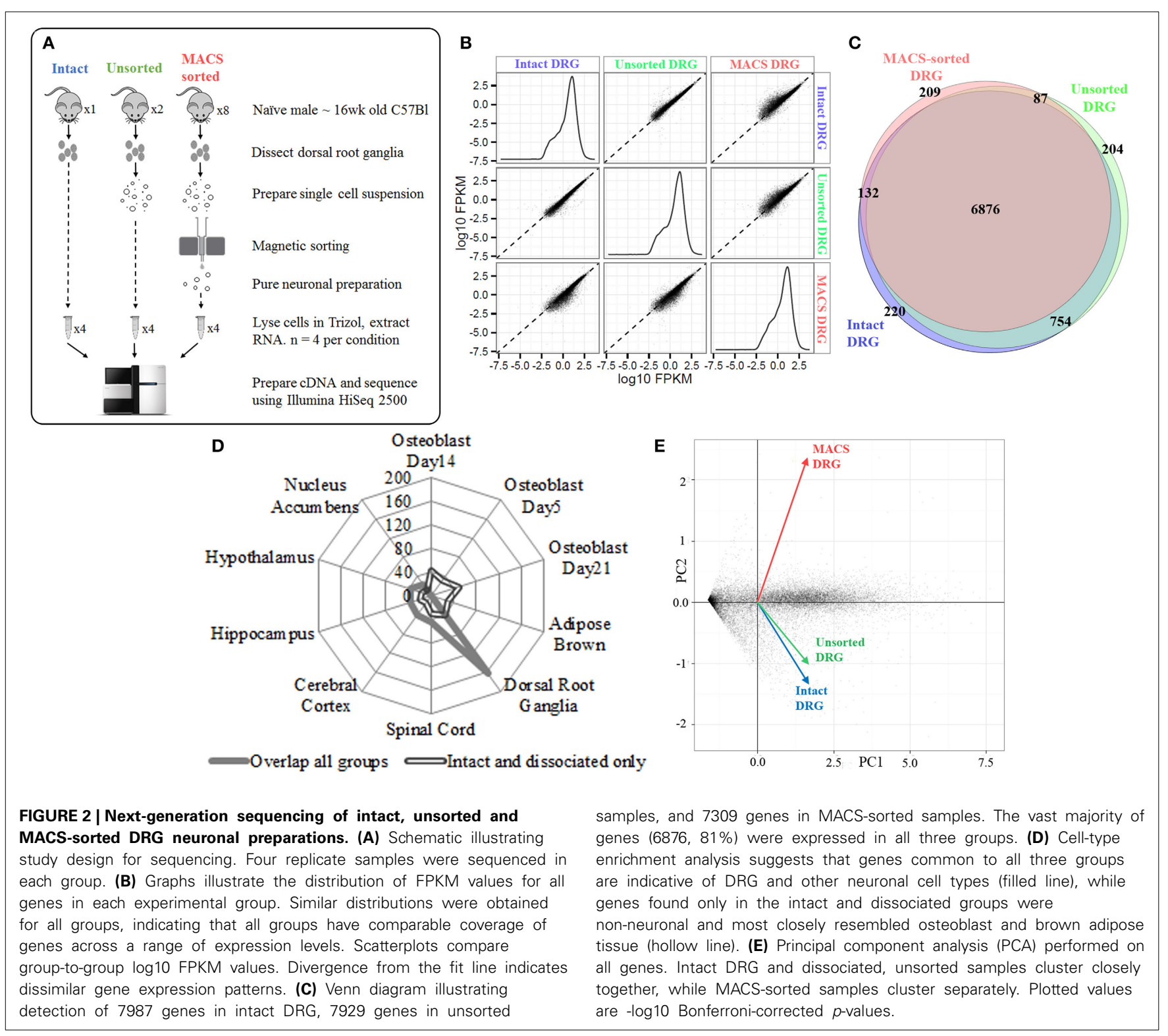

\section{DIFFERENTIAL SPLICING OF THE TrkB RECEPTOR IN NEURONS AND NON-NEURONAL CELLS}

One major advantage of RNA-Sequencing over microarrays is the ability to measure isoform-level differences between samples. Using Cuffdiff we identified 2444 transcripts that were differentially expressed between MACS-sorted samples and unsorted DRG samples, of which 1473 were depleted and 971 were enriched in nociceptors, as well as 99 genes with alternate promoter use and 63 genes that were differentially spliced (Supplementary Table 4). Interestingly, one of the genes that was significantly differentially expressed at the transcript level was the brain-derived nerve growth factor (BDNF) receptor, Ntrk2 (TrkB). There are two isoforms of mouse Ntrk2: one (NM_001025074) encodes the full-length receptor and the other (NM_008745) encodes a truncated version. Although both isoforms were depleted from MACS cultures, the truncated form was downregulated to a much greater extent ( $\log 2$-fold change $=-2.9,=-6.9$, Figure 4$)$ and the message was virtually absent (mean FPKM $=0.23$, s.e.m. $=0.12$, Figure 4 and Supplementary Table 4). This was similar to what was observed for known non-neuronal genes in the MACS-sorted samples, for example the voltage gated potassium channel Kir4.1 (Kcnj10, FPKM $=0.15$, Supplementary Table 1 ) and the glutamate transporter GLAST-1 (Slcla3 FPKM $=0.04$, Supplementary Table 1). This suggests that, in contrast to CNS tissues where the truncated form is expressed in both neuronal and nonneuronal cells, in DRG truncated TrkB is primarily expressed in non-neuronal cells while neurons only express the full length isoform.

Isoform-specific expression analyses were also performed against cortical neurons. These results may be accessed in Supplementary Table 5. 


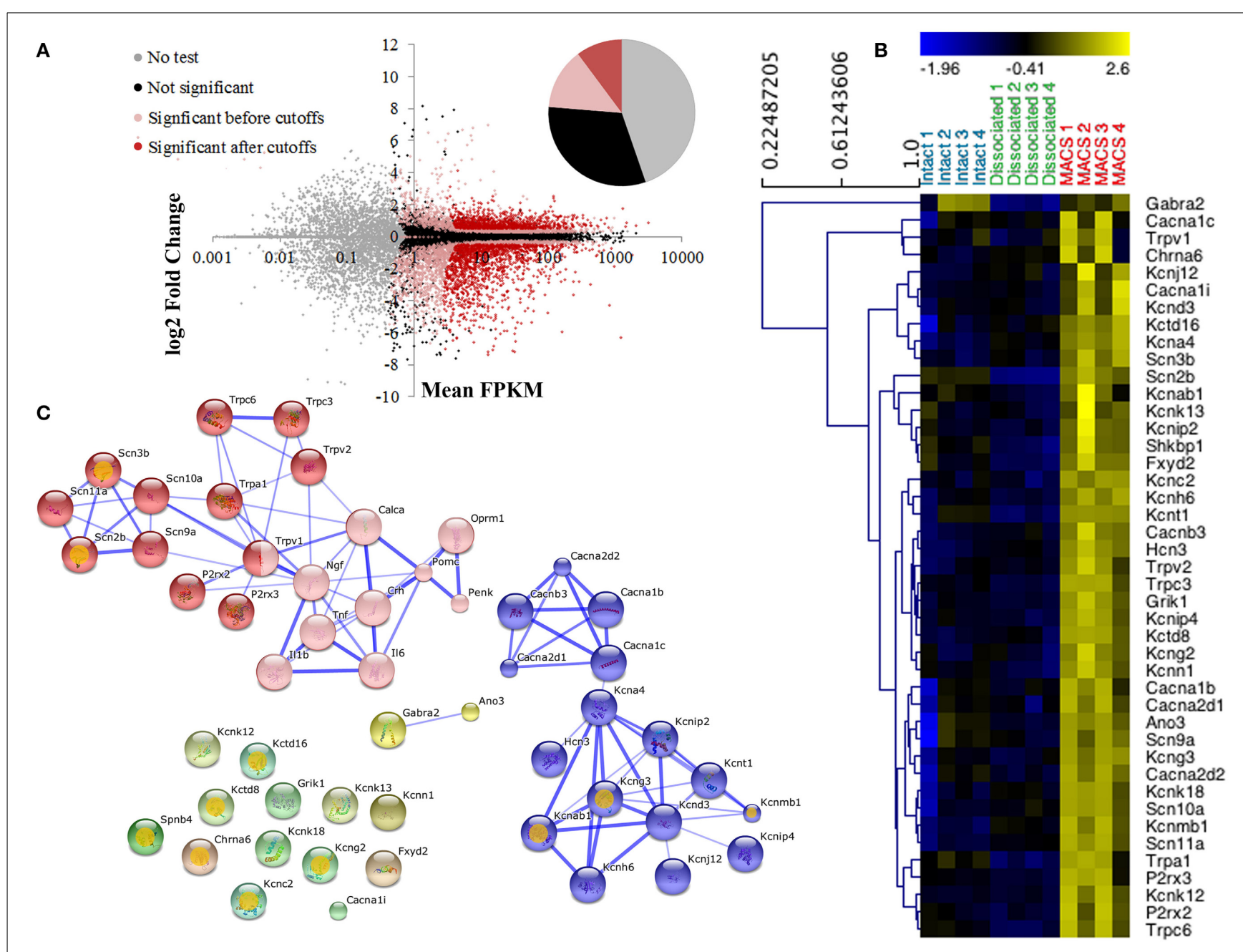

FIGURE 3 | Differential expression profiling of unsorted and MACS-sorted DRG cultures. (A) Cuffdiff was used for differential expression testing between unsorted and MACS-sorted samples. Individual points represent genes. Of 23,991 annotated genes, 10,724 $(44.7 \%)$ were not tested due to their low expression levels. Of the remaining genes, $7579(31.6 \%)$ were not significantly differentially expressed and 5688 (23.7\%) were. Of these, 2443 (10.2\% of total) passed stringent fold change and expression criteria and were used for downstream analysis. (B) Heat map of ion channel expression in intact DRG, unsorted and MACS-sorted cultures. Genes are hierarchically clustered using average linkage clustering and expression is normalized by gene. Ion channels were identified by functional annotation enrichment analysis of MACS genes as they were found to be the most enriched gene class (GO term "ion channel activity," $p=1.89 \mathrm{E}-10, \mathrm{FDR}=$ 2.80E-07) (C) Network analysis of ion channel genes and pain-associated genes. Pink nodes represent pain-associated genes from the pain interactome (Jamieson et al., 2014); orange spots indicate genes with no known pain association; thickness of the edge represents the confidence of the interaction. Unbiased MCL clustering revealed two primary sub-clusters of ion channel genes, the majority of which have been previously studied in the context of pain. Many of the ion channels that do not group into these clusters are novel targets in the context of pain.

\section{MACS-SORTING IDENTIFIES NOVEL TRANSCRIPTION FACTORS PRESENT IN NOCICEPTORS}

Transcriptional regulation is an important mechanism for chronic pain (Perkins et al., 2014). Functional annotation analysis of nociceptor-enriched genes identified 70 genes associated with nucleic acid binding and transcription factor activity (the top 15 are presented in Table 2, below, with the full list in Supplementary Figure 3). This list includes Tlx3 and Pou4f1, genes that are known to be critical for sensory neuron development (Lopes et al., 2012; Zou et al., 2012) as well as 49 genes whose role in pain and somatosensation is not yet known. Unbiased network analysis indicates that among these newly identified nociceptor transcription factors, Klf5, Med10, Myc, and Stat6 are predicted to interact with key nodes of the pain interactome (Supplementary Figure 4; Jamieson et al., 2014).

Importantly, comparison of intact DRG vs. dissociated DRG samples indicated that only two of these genes were found to be significantly induced after dissociation, though fold changes were small $(q<0.05, Z f h x 3 \log 2 \mathrm{fc}=0.39$, Sox4 log $2 \mathrm{fc}=0.45$, Supplementary Table 6). This implies that this list does not represent de novo induction of transcription factor expression as a result of cell extraction and sorting, but rather reflects enrichment in nociceptors. 


\begin{tabular}{|c|c|c|c|}
\hline Gene name & Full gene description & $\begin{array}{l}\text { Fold change } \\
\text { MACS vs. } \\
\text { dissociated } \\
\text { DRG }\end{array}$ & $\begin{array}{l}\text { Fold change } \\
\text { MACS vs. } \\
\text { cortical } \\
\text { neurons }\end{array}$ \\
\hline Calcb & $\begin{array}{l}\text { Calcitonin-related } \\
\text { polypeptide beta }\end{array}$ & 1.46 & 487.22 \\
\hline Mrgpra2b & $\begin{array}{l}\text { MAS-related GPR, } \\
\text { member A2B }\end{array}$ & 1.87 & inf \\
\hline Mrgpra3 & $\begin{array}{l}\text { MAS-related GPR, } \\
\text { member A3 }\end{array}$ & 4.42 & inf \\
\hline Mrgprb4 & $\begin{array}{l}\text { MAS-related GPR, } \\
\text { member B4 }\end{array}$ & 1.86 & inf \\
\hline Mrgprb5 & $\begin{array}{l}\text { MAS-related GPR, } \\
\text { member B5 }\end{array}$ & 3.25 & inf \\
\hline Mrgprd & $\begin{array}{l}\text { MAS-related GPR, } \\
\text { member D }\end{array}$ & 2.01 & inf \\
\hline Mrgpre & $\begin{array}{l}\text { MAS-related GPR, } \\
\text { member E }\end{array}$ & 1.48 & 5.63 \\
\hline Mrgprx1 & $\begin{array}{l}\text { MAS-related GPR, } \\
\text { member X1 }\end{array}$ & 3.19 & $\inf$ \\
\hline$P 2 r \times 2$ & Purinoreceptor 2 & 3.36 & 4.95 \\
\hline$P 2 r \times 3$ & Purinoreceptor 3 & 1.90 & 695.23 \\
\hline P2ry1 & $\begin{array}{l}\text { Purinergic receptor } \\
\text { P2Y, G-protein } \\
\text { coupled, } 1\end{array}$ & 2.51 & 7.37 \\
\hline Prph & Peripherin & 1.80 & 1189.14 \\
\hline Ret & RET (GDNF receptor) & 1.74 & 190.11 \\
\hline Scn10a & $\mathrm{Na}_{v} 1.8$ & 1.71 & 8010.07 \\
\hline Scn11a & $\mathrm{Na}_{v} 1.9$ & 2.14 & 3084.88 \\
\hline Scn9a & $\mathrm{Na}_{\vee} 1.7$ & 1.47 & 38.76 \\
\hline Sst & Somatostatin & 2.41 & 0.02 \\
\hline Tac1 & $\begin{array}{l}\text { Protachykinin-1 } \\
\text { (Substance P } \\
\text { precursor) }\end{array}$ & 1.53 & 24.97 \\
\hline Trpa1 & TRPA1 & 1.83 & 176.05 \\
\hline Trpv1 & TRPV1 & 1.47 & 50.21 \\
\hline Trpv2 & TRPV2 & 1.56 & 5.44 \\
\hline
\end{tabular}

Many of these genes are also enriched in MACS-sorted nociceptors compared to cortical neurons, indicating that these genes are not only nociceptor-enriched, but may in fact be nociceptor-specific (see also Supplementary Figure 2 and Table 2).

\section{DISCUSSION}

We present the first application of magnetic cell sorting (MACS) to isolate and characterize nociceptor neurons from the adult DRG using RNA-sequencing. We find that MACS increases the purity of DRG sensory neuron cell body preparations from $<10 \%$ to $>95 \%$, a purity that cannot be obtained through any other method. These MACS preparations are enriched for nociceptor neurons $<30 \mu \mathrm{m}$ in size expressing the voltage gated sodium channel, $\mathrm{Na}_{\mathrm{v}} 1.8$. Comparison of RNA-sequencing data in unsorted and nociceptor-enriched MACS-sorted preparations enables genome-wide resolution of the nociceptor transcriptome.
We present novel biological insight into the ion channels, growth factor receptor isoforms and transcription factors present in these cells, and anticipate that both the novel sorting technique used and the data generated will be of great utility to researchers studying nociceptors in the context of pain, injury and regeneration.

The importance of attaining cell-type specificity in the context of high-throughput transcriptomic technologies (most often microarray and RNA-sequencing) is becoming increasingly clear (as reviewed in Okaty et al., 2011a; Cruz et al., 2013). Data generated from central nervous system (CNS) or peripheral nervous system (PNS) tissue, given the high level of heterogeneity, is subject to a number of limitations. The first is that any transcriptional change occurring in small populations of cells is likely to be underestimated if assessed in a composite tissue. Likewise, genes regulated in opposite directions in neighboring cell types will appear static in composite data. Similarly, transcripts which are differentially spliced in different cell types cannot be distinguished. Furthermore, following experimental manipulations such as injury, any change in the population of cells present in the tissue (such as infiltration of immune cells) complicates the attribution of any observed transcriptional change.

Obtaining cell-type specificity requires a means of identifying, and a means of isolating cells of interest. A number of features of adult neural tissue limit the applicability of the most common high throughput sorting technique, fluorescence-activated cell sorting (FACS), in PNS. The sensitivity of adult neuronal cell bodies to physical stress, the requirement for enzymatic disruption of tissue that may affect extracellular antibody epitopes, and the relatively small number of cells available make it difficult to recover sufficient RNA for high-throughput applications when flow sorting PNS tissue.

Another sorting technique that is suitable for transcriptome analysis of neural tissue is Translating Ribosome Affinity Purification (TRAP)-Seq. TRAP requires a transgenic mouse with a cell-type specific reporter allowing the labeling and isolation of ribosome-associated RNA within the cells of interest. One strength of the technique is that cell-type specific data can be pulled down from a composite tissue without having to separate constituent cell types. However, data generated using this technique does appear to be subject to a high degree of contamination from other cell types (Okaty et al., 2011b). Furthermore, TRAP is entirely dependent on the fidelity of the cell-type reporter used, and, in contrast to RNA-sequencing of whole-cell lysate, cannot detect non-coding RNA species.

Both FACS and TRAP require a transgenic reporter with high fidelity for the cell type of interest, and are not applicable to non-transgenic animals. In contrast, the MACS-sorting technique can generate pure neuronal preparations from standard laboratory animals with sufficient yield to carry out genome-wide sequencing.

Aside from transcriptomic studies, MACS cultures will also be extremely useful for in vitro study. Previously, preparing an enriched preparation of DRG neurons required extended culture ( $>1$ week) in the presence of a mitotic inhibitor to deplete contaminating non-neuronal cells (Lerch et al., 2012). This culture procedure is not widely used because of concerns about off-target effects of mitotic inhibitors on neurons (Wallace and 


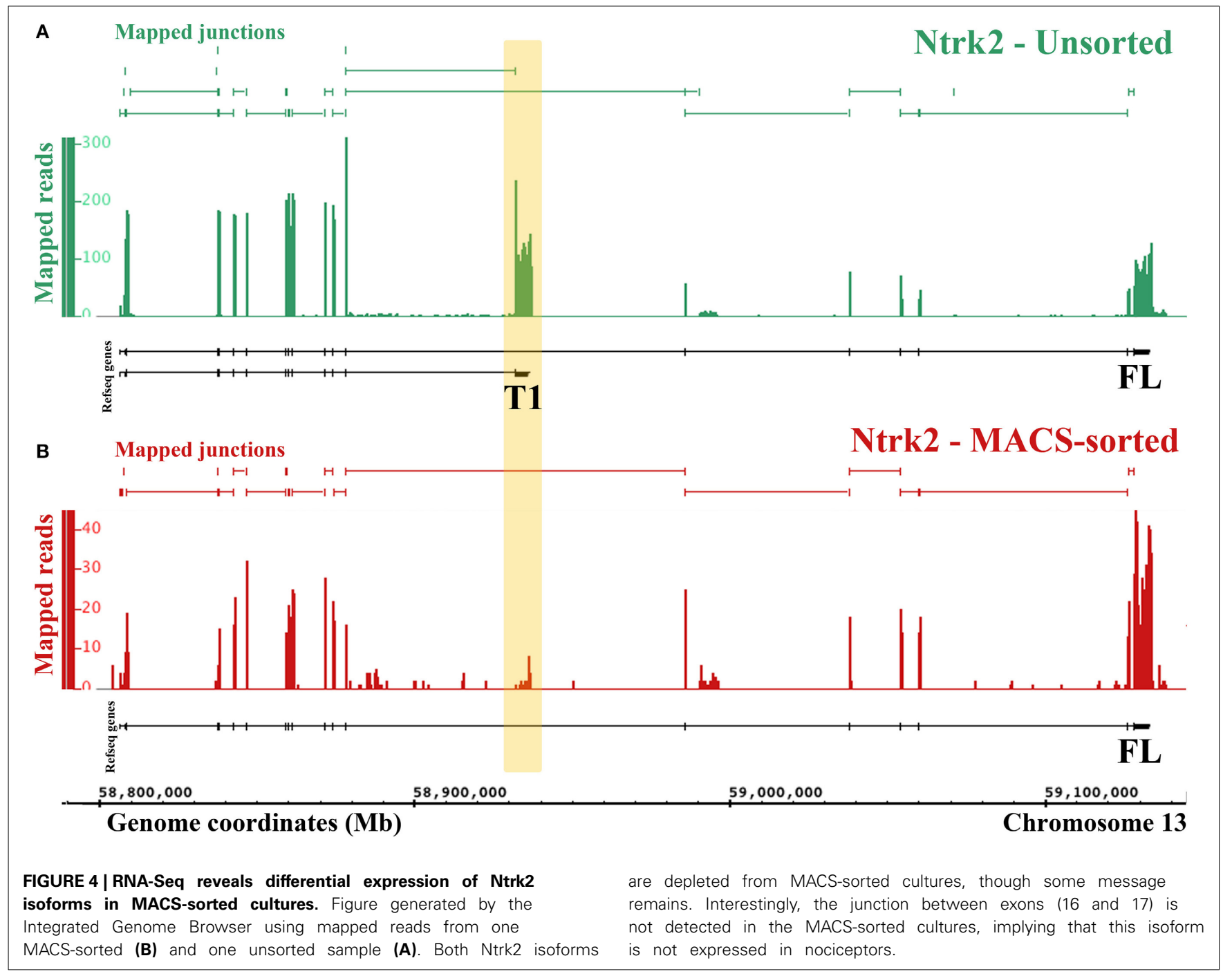

Table 2 | The most highly enriched genes with nucleic acid binding functions present in nociceptors.

\begin{tabular}{|c|c|c|c|c|}
\hline Gene symbol & Full gene name & FPKM unsorted & FPKM MACS & log2-fold change \\
\hline KIf5 & Krueppel-like factor 5 & 14.20 & 43.55 & 1.62 \\
\hline Sox11 & SRY-box 11 & 2.30 & 5.55 & 1.27 \\
\hline Pou4f2 & POU domain class 4 transcription factor 2/Brn3b & 7.57 & 18.23 & 1.27 \\
\hline Pdlim1 & PDZ and LIM domain protein 1 & 6.50 & 15.58 & 1.26 \\
\hline Atf5 & Activating transcription factor 5 & 32.35 & 75.84 & 1.23 \\
\hline Is/2 & Islet 2 & 81.72 & 190.99 & 1.22 \\
\hline$T / x 3$ & T-cell leukemia homeobox 3 & 38.59 & 86.16 & 1.16 \\
\hline Myt1 & Myelin transcription factor 1 & 15.02 & 33.49 & 1.16 \\
\hline Grh/3 & Grainyhead-like 3 & 3.73 & 8.26 & 1.15 \\
\hline Casz1 & Castor zinc-finger 1 & 14.45 & 31.99 & 1.15 \\
\hline
\end{tabular}

Genes already known to have a role in these cells are highlighted in bold. 
Johnson, 1989), the likelihood that DRG neuron phenotype following prolonged culture will no longer resemble that of intact DRG (Buschmann et al., 1998) and the low purity of the culture it produces [74\% neuronal (Lerch et al., 2012)]. For these reasons, most investigators simply use mixed cultures when studying DRG. However, for experiments focused on growth factor signaling, where contaminating non-neuronal cells are sensitive to and able to release growth factors, impurity of DRG culture is a significant limitation (Kalous et al., 2012). The ability to generate pure neuronal cultures will significantly simplify interpretation of in vitro experiments using dissociated DRG neurons.

Our study design allowed us to identify nociceptor-enriched genes by comparing global gene expression profiles of MACSsorted small DRG neurons and dissociated whole ganglia. Further in silico analysis to compare nociceptor and cortical neuron transcriptomes revealed that the majority of nociceptor-enriched genes may also be PNS-specific, as these were found to be significantly upregulated compared to cortical neurons. These genes may therefore be useful drivers for nociceptor-specific Cre lines, or for PNS-specific drug targeting. Further investigation of other PNS neuronal subtypes, for example autonomic ganglia, would help to refine this list.

One limitation of our study is that we cannot definitively determine the source of genes that are expressed at lower levels in nociceptors compared to the whole DRG. This is because these transcripts could come from either large neurons or nonneuronal cells. However, we observed that known non-neuronal genes tend to have very low expression levels in MACS sorted cultures (FPKM < 0.5). We used this heuristic to interpret the alternate Ntrk2 isoform expression (Figure 4). Further work to isolate and transcriptionally profile all of the cell types of the DRG are required to verify the origin of depleted transcripts. However, in the absence of this information, the list of depleted genes may still be used to inform experiments. If researchers are interested in a particular receptor due to pharmacological efficacy, our differential expression data will help to identify whether results may be due to a direct action on nociceptors (i.e. if the receptor is enriched), or an indirect action (if the receptor is not present).

Many of the ion channels we identify in nociceptors (including Kcnab1, Scn2, and Kcnc2) have not previously been described in the PNS. Given the attractiveness of therapeutics targeting ion channels such as the sodium channel $\mathrm{Na}_{\mathrm{v}} 1.7$ (Eijkelkamp et al., 2012; Lee et al., 2014), these channels could constitute novel targets for the treatment of pain or itch. One interesting feature of the nociceptor transcriptome we observe is the relatively low enrichment of TRPV1 $\left(\log _{2} \mathrm{FC}=0.59\right)$ in the MACS-sorted preparation. This likely reflects the size-selection of the MACS method, which will exclude TRPV1+ A-fibers. This results in a relatively low enrichment for TRPV1 in the MACS-sorted preparation relative to unsorted DRG, as the latter contains these TRPV1+ A-fibers (Mitchell et al., 2010).

The nociceptor-enriched transcription factors we describe include a number with known roles in DRG morphogenesis and function. These include $T l \times 3$ and Pou 4 f, which control the development of subsets of nociceptors (Lopes et al., 2012; Zou et al., 2012). Disrupting expression of nociceptor development-controlling transcription factors can be incredibly informative-for example, disruption of Runxl reveals the genes uniquely expressed in cutaneous and deep-tissue innervating nociceptors (Yang et al., 2013), while Runx3 can be used to disrupt development of DRG proprioceptive neurons peripheral and central axonal projections (Inoue et al., 2002; Levanon et al., 2002).

Of the 70 genes with nucleic acid binding activity we identify in nociceptors, only 21 have a known function in DRG. Of these 21 previously characterized genes, many control neurite outgrowth and axonal regeneration following injury, including Sox11, Pdlim1, Nfil3, Hivep3, and Smad1 (Jankowski et al., 2006; Wu et al., 2006; Macgillavry et al., 2009; Ohno et al., 2009; Zou et al., 2009). The remaining 49 uncharacterized genes include Pou 42 and Atf5, genes involved in the acute response to damage and axon outgrowth in the optic nerve, another popular model tissue for regeneration (Huang et al., 2014; Yasuda et al., 2014).

The finding of differential expression of the full length (FL) and truncated (T1) isoforms of the BDNF receptor TrkB suggests for the first time that only TrkB FL is present in nociceptors, with TrkB T1 expressed in non-neuronal cells of the DRG (Schwann cells or satellite glia). This advances previous studies in the CNS, where TrkB T1 was found in glia, but both forms were detected in neurons (Frisen et al., 1993; Armanini et al., 1995). The nociceptor transcriptome we present allows investigators to assess transcript-level differential expression for any gene of interest.

In summary, the DRG nociceptor preparation produced by MACS-sorting has many applications in vitro and in molecular studies. We have demonstrated how the nociceptor transcriptome, generated via RNA-sequencing of MACS-sorted DRG, can be mined to gain novel biological insight. Knowledge of the ion channels, splice isoforms and transcription factors enriched in nociceptors will be of great utility to investigators studying this tissue in the context of pain, somatosensation and itch. As sequencing data in a wider array of other tissues and cellular subtypes becomes available, it will deepen our understanding of the defining features of the nociceptor transcriptome.

\section{AUTHOR CONTRIBUTIONS}

Data acquisition and analysis Matthew Thakur, Megan Crow, Natalie Richards, Gareth I. J. Davey, Emma Levine, Jayne H. Kelleher, Chibeza C. Agley, Stephen D. R. Harridge. Analysis of sequencing data/Bioinformatics Megan Crow, Franziska Denk. Drafting of manuscript Matthew Thakur, Megan Crow. Editing of manuscript Matthew Thakur, Megan Crow, Franziska Denk, Stephen B. McMahon.

\section{ACKNOWLEDGMENTS}

We thank the High Throughput Genomics Group at the Wellcome Trust Centre for Human Genetics (funded by Wellcome Trust grant references 090532/Z/09/Z and MRC Hub grant G0900747 91070) for the generation of sequencing data. Cytometry facilities were provided by the National Institute for Health Research (NIHR) Biomedical Research Centre based at Guy's and St Thomas' NHS Foundation Trust and King's College 
London. The views expressed are those of the author(s) and not necessarily those of the NHS, the NIHR or the Department of Health. We also thank Prof John Wood at UCL for his gift of $\mathrm{Na}_{\mathrm{v}} 1.8$ TdTom mice.

\section{SUPPLEMENTARY MATERIAL}

The Supplementary Material for this article can be found online at: http://www.frontiersin.org/journal/10.3389/fnmol. 2014.00087/abstract

\section{REFERENCES}

An, X., Schulz, V. P., Li, J., Wu, K., Liu, J., Xue, F., et al. (2014). Global transcriptome analyses of human and murine terminal erythroid differentiation. Blood 123, 3466-3477. doi: 10.1182/blood-2014-01548305

Armanini, M. P., McMahon, S. B., Sutherland, J., Shelton, D. L., and Phillips, H. S. (1995). Truncated and catalytic isoforms of trkB are co-expressed in neurons of rat and mouse CNS. Eur. J. Neurosci. 7, 1403-1409. doi: 10.1111/j.14609568.1995.tb01132.x

Bauer, C. S., Nieto-Rostro, M., Rahman, W., Tran-Van-Minh, A., Ferron, L., Douglas, L., et al. (2009). The increased trafficking of the calcium channel subunit alpha2delta-1 to presynaptic terminals in neuropathic pain is inhibited by the alpha2delta ligand pregabalin. J. Neurosci. 29, 4076-4088. doi: 10.1523/JNEUROSCI.0356-09.2009

Blankenberg, D., Kuster, G. V., Coraor, N., Ananda, G., Lazarus, R., Mangan, M., et al. (2010). Galaxy: a web-based genome analysis tool for experimentalists. Curr. Protoc. Mol. Biol. 89, 19.10.1-19.10.21. doi: 10.1002/0471142727.mb1910s89

Breivik, H., Eisenberg, E., O'Brien, T., and Openminds. (2013). The individual and societal burden of chronic pain in Europe: the case for strategic prioritisation and action to improve knowledge and availability of appropriate care. BMC Public Health 13:1229. doi: 10.1186/1471-2458-13-1229

Buschmann, T., Martin-Villalba, A., Kocsis, J., Waxman, S., Zimmermann, M., and Herdegen, T. (1998). Expression of Jun, Fos and ATF-2 proteins in axotomized explanted and cultured adult rat dorsal root ganglia. Neuroscience 84, 163-176. doi: 10.1016/S0306-4522(97)00487-9

Cruz, F. C., Koya, E., Guez-Barber, D. H., Bossert, J. M., Lupica, C. R., Shaham, Y., et al. (2013). New technologies for examining the role of neuronal ensembles in drug addiction and fear. Nat. Rev. Neurosci. 14, 743-754. doi: 10.1038/ nrn3597

Da Wei Huang, B. T. S., and Lempicki, R. A. (2008). Systematic and integrative analysis of large gene lists using DAVID bioinformatics resources. Nat. Protoc. 4, 44-57. doi: 10.1038/nprot.2008.211

Eijkelkamp, N., Linley, J. E., Baker, M. D., Minett, M. S., Cregg, R., Werdehausen, R., et al. (2012). Neurological perspectives on voltage-gated sodium channels. Brain 135, 2585-2612. doi: 10.1093/brain/aws225

Frisen, J., Verge, V. M., Fried, K., Risling, M., Persson, H., Trotter, J., et al. (1993). Characterization of glial trkB receptors: differential response to injury in the central and peripheral nervous systems. Proc. Natl. Acad. Sci. U.S.A. 90, 4971-4975. doi: 10.1073/pnas.90.11.4971

Giardine, B., Riemer, C., Hardison, R. C., Burhans, R., Elnitski, L., Shah, P., et al. (2005). Galaxy: a platform for interactive large-scale genome analysis. Genome Res. 15, 1451-1455. doi: 10.1101/gr.4086505

Goecks, J., Nekrutenko, A., and Taylor, J. (2010). Galaxy: a comprehensive approach for supporting accessible, reproducible, and transparent computational research in the life sciences. Genome Biol. 11:R86. doi: 10.1186/gb-201011-8-r86

Heng, T. S., Painter, M. W., and Immunological Genome Project, C. (2008). The Immunological Genome Project: networks of gene expression in immune cells. Nat. Immunol. 9, 1091-1094. doi: 10.1038/ni1008-1091

Hu, M., Yao, J., Cai, L., Bachman, K. E., Van Den Brule, F., Velculescu, V., et al. (2005). Distinct epigenetic changes in the stromal cells of breast cancers. Nat. Genet. 37, 899-905. doi: 10.1038/ng1596

Huang, D. W., Sherman, B. T., and Lempicki, R. A. (2009). Bioinformatics enrichment tools: paths toward the comprehensive functional analysis of large gene lists. Nucleic Acids Res. 37, 1-13. doi: 10.1093/nar/gkn923
Huang, L., Hu, F., Xie, X., Harder, J., Fernandes, K., Zeng, X.-Y., et al. (2014). Pou4f1 and pou4f 2 are dispensable for the long-term survival of adult retinal ganglion cells in mice. PLoS ONE 9:e94173. doi: 10.1371/journal.pone.0094173

Inoue, K.-I., Ozaki, S., Shiga, T., Ito, K., Masuda, T., Okado, N., et al. (2002). Runx3 controls the axonal projection of proprioceptive dorsal root ganglion neurons. Nat. Neurosci. 5, 946-954. doi: 10.1038/nn925

Jamieson, D. G., Moss, A., Kennedy, M., Jones, S., Nenadic, G., Robertson, D. L., et al. (2014). The pain interactome: connecting pain specific protein interactions. Pain. doi: 10.1016/j.pain.2014.06.020. [Epub ahead of print].

Jankowski, M. P., Cornuet, P. K., McIlwrath, S., Koerber, H. R., and Albers, K. M. (2006). SRY-box containing gene 11 (Sox11) transcription factor is required for neuron survival and neurite growth. Neuroscience 143, 501-514. doi: 10.1016/j.neuroscience.2006.09.010

Jensen, L. J., Kuhn, M., Stark, M., Chaffron, S., Creevey, C., Muller, J., et al. (2009). STRING 8-a global view on proteins and their functional interactions in 630 organisms. Nucleic Acids Res. 37, D412-D416. doi: 10.1093/nar/gkn760

Kalous, A., Nangle, M. R., Anastasia, A., Hempstead, B. L., and Keast, J. R. (2012). Neurotrophic actions initiated by proNGF in adult sensory neurons may require peri-somatic glia to drive local cleavage to NGF. J. Neurochem. 122, 523-536. doi: 10.1111/j.1471-4159.2012.07799.x

Kim, D., Pertea, G., Trapnell, C., Pimentel, H., Kelley, R., and Salzberg, S. L. (2013). TopHat2: accurate alignment of transcriptomes in the presence of insertions, deletions and gene fusions. Genome Biol. 14:R36. doi: 10.1186/gb-201314-4-r36

Lee, J.-H., Park, C.-K., Chen, G., Han, Q., Xie, R.-G., Liu, T., et al. (2014). A monoclonal antibody that targets a $\mathrm{Na}_{\mathrm{v}} 1.7$ channel voltage sensor for pain and itch relief. Cell 157, 1393-1404. doi: 10.1016/j.cell.2014.03.064

Lerch, J. K., Kuo, F., Motti, D., Morris, R., Bixby, J. L., and Lemmon, V. P. (2012). Isoform diversity and regulation in peripheral and central neurons revealed through RNA-Seq. PLoS ONE 7:e30417. doi: 10.1371/journal.pone. 0030417

Levanon, D., Bettoun, D., Harris-Cerruti, C., Woolf, E., Negreanu, V., Eilam, R., et al. (2002). The Runx3 transcription factor regulates development and survival of TrkC dorsal root ganglia neurons. EMBO J. 21, 3454-3463. doi: 10.1093/emboj/cdf370

Li, H., Handsaker, B., Wysoker, A., Fennell, T., Ruan, J., Homer, N., et al. (2009). The sequence alignment/map format and SAMtools. Bioinformatics 25, 2078-2079. doi: 10.1093/bioinformatics/btp352

Lobo, M. K., Karsten, S. L., Gray, M., Geschwind, D. H., and Yang, X. W. (2006). FACS-array profiling of striatal projection neuron subtypes in juvenile and adult mouse brains. Nat. Neurosci. 9, 443-452. doi: 10.1038/ nn 1654

Lopes, C., Liu, Z., Xu, Y., and Ma, Q. (2012). Tlx3 and Runx1 act in combination to coordinate the development of a cohort of nociceptors, thermoceptors, and pruriceptors. J. Neurosci. 32, 9706-9715. doi: 10.1523/JNEUROSCI.110912.2012

Macgillavry, H. D., Stam, F. J., Sassen, M. M., Kegel, L., Hendriks, W. T., Verhaagen, J., et al. (2009). NFIL3 and cAMP response element-binding protein form a transcriptional feedforward loop that controls neuronal regeneration-associated gene expression. J. Neurosci. 29, 15542-15550. doi: 10.1523/JNEUROSCI.3938-09.2009

Malin, S. A., Davis, B. M., and Molliver, D. C. (2007). Production of dissociated sensory neuron cultures and considerations for their use in studying neuronal function and plasticity. Nat. Protoc. 2, 152-160. doi: 10.1038/nprot. 2006.461

Mi, H., Muruganujan, A., and Thomas, P. D. (2013). PANTHER in 2013: modeling the evolution of gene function, and other gene attributes, in the context of phylogenetic trees. Nucleic Acids Res. 41, D377-D386. doi: 10.1093/nar/ gks1118

Mitchell, K., Bates, B. D., Keller, J. M., Lopez, M., Scholl, L., Navarro, J., et al. (2010). Ablation of rat TRPV1-expressing Adelta/C-fibers with resiniferatoxin: analysis of withdrawal behaviors, recovery of function and molecular correlates. $\mathrm{Mol}$. Pain 6, 8069-8066. doi: 10.1186/1744-8069-6-94

Ohno, K., Kato, H., Funahashi, S., Hasegawa, T., and Sato, K. (2009). Characterization of CLP36/Elfin/PDLIM1 in the nervous system. J. Neurochem. 111, 790-800. doi: 10.1111/j.1471-4159.2009.06370.x

Okaty, B. W., Sugino, K., and Nelson, S. B. (2011a). Cell type-specific transcriptomics in the brain. J. Neurosci. 31, 6939-6943. doi: 10.1523/JNEUROSCI.062611.2011 
Okaty, B. W., Sugino, K., and Nelson, S. B. (2011b). A quantitative comparison of cell-type-specific microarray gene expression profiling methods in the mouse brain. PLoS ONE 6:e16493. doi: 10.1371/journal.pone.0016493

Perkins, J. R., Antunes-Martins, A., Calvo, M., Grist, J., Rust, W., Schmid, R., et al. (2014). A comparison of RNA-seq and exon arrays for whole genome transcription profiling of the L5 spinal nerve transection model of neuropathic pain in the rat. Mol. Pain 10:7. doi: 10.1186/1744-8069-10-7

Saeed, A., Sharov, V., White, J., Li, J., Liang, W., Bhagabati, N., et al. (2003). TM4: a free, open-source system for microarray data management and analysis. Biotechniques 34, 374-378.

Shields, S. D., Ahn, H.-S., Yang, Y., Han, C., Seal, R. P., Wood, J. N., et al. (2012). $\mathrm{Na}_{\mathrm{v}} 1.8$ expression is not restricted to nociceptors in mouse peripheral nervous system. Pain 153, 2017-2030. doi: 10.1016/j.pain.2012. 04.022

Shoemaker, J. E., Lopes, T. J., Ghosh, S., Matsuoka, Y., Kawaoka, Y., and Kitano, H. (2012). CTen: a web-based platform for identifying enriched cell types from heterogeneous microarray data. BMC Genomics 13:460. doi: 10.1186/14712164-13-460

Trapnell, C., Roberts, A., Goff, L., Pertea, G., Kim, D., Kelley, D. R., et al. (2012). Differential gene and transcript expression analysis of RNA-seq experiments with TopHat and Cufflinks. Nat. Protoc. 7, 562-578. doi: 10.1038/nprot.2012.016

Wallace, T. L., and Johnson, E. M. (1989). Cytosine arabinoside kills postmitotic neurons: evidence that deoxycytidine may have a role in neuronal survival that is independent of DNA synthesis. J. Neurosci. 9, 115-124.

Wu, L. C., Goettl, V. M., Madiai, F., Hackshaw, K. V., and Hussain, S. R. (2006). Reciprocal regulation of nuclear factor kappa B and its inhibitor ZAS3 after peripheral nerve injury. BMC Neurosci. 7:4. doi: 10.1186/1471-2202-7-4

Yang, F.-C., Tan, T., Huang, T., Christianson, J., Samad, O. A., Liu, Y., et al. (2013). Genetic control of the segregation of pain-related sensory neurons innervating the cutaneous versus deep tissues. Cell Rep. 5, 1353-1364. doi: 10.1016/j.celrep.2013.11.005
Yasuda, M., Tanaka, Y., Ryu, M., Tsuda, S., and Nakazawa, T. (2014). RNA sequence reveals mouse retinal transcriptome changes early after axonal injury. PLoS ONE 9:e93258. doi: 10.1371/journal.pone.0093258

Zhang, Y., Chen, K., Sloan, S. A., Bennett, M. L., Scholze, A. R., O’Keeffe, S., et al. (2014). An RNA-sequencing transcriptome and splicing database of glia, neurons, and vascular cells of the cerebral cortex. J. Neurosci. 34, 11929-11947. doi: 10.1523/JNEUROSCI.1860-14.2014

Zou, H., Ho, C., Wong, K., and Tessier-Lavigne, M. (2009). Axotomy-induced Smadl activation promotes axonal growth in adult sensory neurons. J. Neurosci. 29, 7116-7123. doi: 10.1523/JNEUROSCI.5397-08.2009

Zou, M., Li, S., Klein, W. H., and Xiang, M. (2012). Brn3a/Pou4f1 regulates dorsal root ganglion sensory neuron specification and axonal projection into the spinal cord. Dev. Biol. 364, 114-127. doi: 10.1016/j.ydbio.2012. 01.021

Conflict of Interest Statement: The authors declare that the research was conducted in the absence of any commercial or financial relationships that could be construed as a potential conflict of interest.

Received: 02 September 2014; accepted: 25 October 2014; published online: 11 November 2014.

Citation: Thakur M, Crow M, Richards N, Davey GIJ, Levine E, Kelleher JH, Agley CC, Denk F, Harridge SDR and McMahon SB (2014) Defining the nociceptor transcriptome. Front. Mol. Neurosci. 7:87. doi: 10.3389/fnmol.2014.00087 This article was submitted to the journal Frontiers in Molecular Neuroscience. Copyright (C) 2014 Thakur, Crow, Richards, Davey, Levine, Kelleher, Agley, Denk, Harridge and McMahon. This is an open-access article distributed under the terms of the Creative Commons Attribution License (CC BY). The use, distribution or reproduction in other forums is permitted, provided the original author(s) or licensor are credited and that the original publication in this journal is cited, in accordance with accepted academic practice. No use, distribution or reproduction is permitted which does not comply with these terms. 